\title{
First-principles calculations of hyperfine interaction, binding energy, and quadrupole coupling for shallow donors in silicon
}

\author{
Michael W. Swift $\mathbb{D}^{1,2}$, Hartwin Peelaers $\mathbb{D}^{3,4}$, Sai Mu $\mathbb{D}^{3}$, John J. L. Morton ${ }^{5}$ and Chris G. Van de Walle $\mathbb{i D}^{3 凶}$
}

Spin qubits based on shallow donors in silicon are a promising quantum information technology with enormous potential scalability due to the existence of robust silicon-processing infrastructure. However, the most accurate theories of donor electronic structure lack predictive power because of their reliance on empirical fitting parameters, while predictive ab initio methods have so far been lacking in accuracy due to size of the donor wavefunction compared to typical simulation cells. We show that density functional theory with hybrid and traditional functionals working in tandem can bridge this gap. Our first-principles approach allows remarkable accuracy in binding energies ( $67 \mathrm{meV}$ for bismuth and $54 \mathrm{meV}$ for arsenic) without the use of empirical fitting. We also obtain reasonable hyperfine parameters (1263 MHz for Bi and $133 \mathrm{MHz}$ for As) and superhyperfine parameters. We demonstrate the importance of a predictive model by showing that hydrostatic strain has much larger effect on the hyperfine structure than predicted by effective mass theory, and by elucidating the underlying mechanisms through symmetry analysis of the shallow donor charge density.

npj Computational Materials (2020)6:181 ; https://doi.org/10.1038/s41524-020-00448-7

\section{INTRODUCTION}

The advent of quantum computers capable of completing tasks beyond the capabilities of the most powerful classical supercomputers represents a new era in computation ${ }^{1}$. In the emerging field of quantum information technology, researchers are exploring a vast array of qubit platforms in a race to build scalable, faulttolerant quantum computers ${ }^{1-6}$. Spin-based qubits leveraging atomic clock transitions of shallow donors in silicon are one such promising technology, exhibiting long coherence times, singlequbit control, and high fidelity ${ }^{7-11}$. Though it still faces many obstacles, an important advantage of this qubit platform is its use of doped silicon, allowing the potential for enormous scalability using existing infrastructure, that has been built up by the semiconductor industry over decades.

Silicon in modern electronics is doped using the same kinds of shallow impurities that make up proposed silicon-based qubits. In the case of $n$-type doping, shallow donors contribute electrons to the conduction-band minimum (CBM), while in $p$-type doping holes are contributed to the valence-band maximum (VBM). In conventional electronic device theory, it is often sufficient to treat the introduced electrons or holes as free carriers in the unperturbed band structure of the host. However, spin-based qubits rely on the detailed electronic structure of the donor state. According to "effective-mass theory" 12 a donor modifies the band occupied by the donated electron much like a point charge modifies the vacuum states. This creates "hydrogenic" states in which the electron is loosely bound to the donor, analogous to the bonding of an electron to a proton in a free hydrogen atom. Originally studied by Kohn and Luttinger in the $1950 \mathrm{~s}^{13-16}$, this model has been extended and improved, but still faces limitations due to its intrinsic approximations. Within hydrogenic effective mass theory, screening of the impurity potential is approximated by using the macroscopic dielectric constant, but actual screening by the valence electrons proceeds in complex material-dependent and impurity-dependent ways. These are known as "central-cell" effects. Further complications arise from the fact that the conduction-band minimum of silicon is a set of six degenerate valleys $^{12}$. These six valleys are not independent, but mix and split through a process known as "valley-orbit coupling". Group theoretic arguments show that the CBM splits into states corresponding to irreducible representations of the $T_{\mathrm{d}}$ symmetry group: singlet $A_{1}$ (the ground state), doublet $E$, and triplet $T_{2}{ }^{17,18}$.

Developments in the treatment of shallow impurities have largely focused on corrections for these effects and attempts to include them in models ${ }^{17-21}$. Within effective mass theory, this effort has met with success largely through the use of empirical fitting parameters. We take a different tack, seeking greater predictive power by calculating properties of shallow impurities from first principles.

The ability to accurately model shallow dopants has taken on particular relevance in the context of quantum information science, as exemplified by the recent experiments of Pla et al. ${ }^{22}$. They used electron spin resonance (ESR) to measure transitions between total-spin states of the coupled electron-nucleus spin degree of freedom of shallow bismuth donors in silicon, a system with applications in atomic clock transitions for silicon-based spin qubits $^{23,24}$. They observed a split Hahn echo peak corresponding to the $|4,-4\rangle \leftrightarrow|5,-5\rangle$ transition. Orientation-dependent measurements suggest that this results from strain caused by the thermal contraction of an aluminum resonator patterned on the surface of the sample. This result raises the question: how does strain cause such a dramatic splitting in the ESR line? Traditional approaches, such as the "valley repopulation model" 25 , fail to provide an explanation. Another model invokes the coupling of the quadrupole moment of the donor nucleus with the electric field gradient (EFG) induced by the electron wavefunction, but accurate information about the strength of this interaction is lacking. $\mathrm{Ab}$ initio calculations have already been shown to

\footnotetext{
'Department of Physics, University of California, Santa Barbara, CA 93106-9530, USA. ²US Naval Research Laboratory, Center for Computational Materials Science, Washington, DC 20375, USA. ${ }^{3}$ Materials Department, University of California, Santa Barbara, CA 93106-5050, USA. ${ }^{4}$ Department of Physics and Astronomy, University of Kansas, Lawrence, Kansas 66045, USA. ${ }^{5}$ Department of Electronic and Electrical Engineering, UCL, London, WC1E 7JE, UK. ${ }^{\bowtie}$ email: vandewalle@mrl.ucsb.edu
} 
successfully determine EFGs at nuclei ${ }^{26}$. An accurate calculation of the donor wavefunction will thus allow the determination of the EFG at the nucleus and the strength of the quadrupole coupling. Additional motivation was provided by subsequent experiments ${ }^{7}$ that identified an unexpectedly large dependency of the isotropic hyperfine parameter $A$ on the hydrostatic component of strain. We will therefore also explicitly explore the strain dependence of $A$.

$\mathrm{Ab}$ initio approaches to donors have met with some success in the past. Density functional theory (DFT) has been shown to correctly predict hyperfine parameters for highly localized defect states ("deep" impurities) 27,28, but explicit calculation of the relatively delocalized shallow states within the supercell approach $^{29}$ is extremely challenging due to the large spatial extent of the wavefunction. Real-space pseudopotential methods have been successful in nanostructures ${ }^{30,31}$. Calculations based on an impurity Green's function approach are in principle more suited, and have been successfully used to study the isotropic hyperfine parameter of shallow donors ${ }^{32}$, to explore their evolution for phosphorus donors at very high $\operatorname{strain}^{33}$, and to calculate superhyperfine parameters ${ }^{34}$. However, the Green's function technique is far less intuitive than the supercell approach, and is difficult to generalize.

Calculations of shallow dopant binding energies have also been attempted through the supercell approach. Wang ${ }^{35}$ used a potential patching method to calculate supercells up to 64,000 atoms, achieving modest success in the calculation of binding energies of some acceptors, and Zhang et al. ${ }^{36}$ improved on this method by incorporating GW corrections based on 64-atom cells, accurately predicting binding energies of various acceptors in $\mathrm{Si}$, GaAs, and GaP. Yamamoto et al. ${ }^{37}$ modeled an As donor in Si by performing DFT calculations using the generalized gradient approximation (GGA) for supercells up to 10,648 atoms. They calculated binding energies based on wavefunctions extracted from the supercells and found a good description of the Bohr radius and the binding energy, but their use of an empirical model for the screened impurity potential sacrifices predictive power. More recently, Smith et al. ${ }^{38}$ used a similar approach for $\mathrm{P}$ in Si. These studies show that, while it is extremely difficult to capture the exponential tail of shallow impurity wavefunctions with firstprinciples methods, DFT may be able to describe the wavefunction correctly in the vicinity of the impurity. This is encouraging because the isotropic hyperfine coupling and quadrupole coupling both depend on the properties of the wavefunction at the donor nucleus. This also suggests that it may be possible to introduce a systematic correction for errors induced by the overlap of the exponential tail of the wavefunction into neighboring supercells.

Even if systematic corrections are applied, the required supercell sizes can only be tackled within DFT by using traditional functionals such as GGA. However, such functionals underestimate electron localization, thus limiting the accuracy of the wavefunction amplitudes in the vicinity of the donor. In this work, we overcome these obstacles by using a tandem approach, judiciously combining GGA results for very large supercells with results obtained with a hybrid functional. which provides a correct description of localization. We are thus able to extrapolate the hybrid functional results to the dilute limit. This technique retains predictive power because it does not use empirical fitting parameters. We will demonstrate the approach using the example of arsenic and bismuth shallow donors in $\mathrm{Si}$, obtaining results that are in excellent agreement with experiment. We also show that the experimentally observed variation of the isotropic hyperfine parameter as a function of strain can be captured in feasible supercell calculations, with central-cell and valley-orbit effects automatically included in the ab initio method.

The present work is on silicon, but the same techniques should work for donors in other multivalley semiconductors such as germanium. Donors in single-valley semiconductors such as gallium arsenide are simpler due to the lack of multivalley effects, and are much better described by effective mass theory ${ }^{17}$. We also expect that our techniques can be generalized to shallow acceptors, including the treatment of the degeneracy at the valence-band maximum.

\section{RESULTS}

Binding energies

The binding energy of a shallow donor may be obtained from the Kohn-Sham eigenvalues:

$E_{\mathrm{b}}=\epsilon_{\Gamma}^{\mathrm{CB}}-\epsilon_{\Gamma}^{\text {donor }}+e \Delta V$,

where $\epsilon_{\Gamma}^{\text {donor }}$ is the Kohn-Sham eigenvalue of the occupied donor state (evaluated at $\Gamma$, as discussed in the "Methods" section) and $\epsilon_{\Gamma}^{\mathrm{CB}}$ is the Kohn-Sham eigenvalue of the CB in a bulk calculation of a supercell of the same size, also evaluated at $\Gamma$ for consistency. Because the Kohn-Sham eigenvalues are referenced to the average electrostatic potential, we must adjust for any shifts in the potential due to the presence of the impurity in order to meaningfully compare eigenvalues between bulk and impurity cells. This is accomplished by introducing the correction term $\Delta V=\left\langle V^{\text {bulk }}-V^{\text {donor }}\right\rangle$, where $\langle\cdot\rangle$ indicates a macroscopic average over a bulk-like region of the cell far from the donor. This term (identical to the potential alignment term used for defect formation energies in the Freysoldt method ${ }^{39,40}$ ) aligns the Kohn-Sham levels between the bulk and donor cells, allowing for meaningful comparison.

Use of the Kohn-Sham eigenvalues rather than DFT total energies is standard for $a b$ initio calculations of donor binding energies ${ }^{35-38}$. Methods based on comparing total energies significantly underestimate $E_{\mathrm{b}}{ }^{35}$.

The quantities used to calculate the binding energy (Eq. (1)) for bismuth and arsenic shallow donors in silicon are given in Table 1, and the resulting binding energies are plotted in Fig. 1. For sufficiently large supercells, the error in the calculated binding energy comes from the exponential tail of the donor wavefunction, which extends into neighboring supercells. This error scales inversely with the volume of the supercell (see the "Methods" section), and therefore we plot our results as a function of $1 / N$ where $N$ is the number of atoms in the supercell. Our results for bismuth donor supercells with $n \geq 4(N \geq 512)$ show a clear linear trend with $1 / N$, allowing extrapolation to the $N \rightarrow \infty$ limit. Extrapolating results obtained with the GGA of Perdew, Burke, and Ernzerhof $^{41}$ (PBE) gives a value of 28.3 meV for bismuth donors, significantly underestimating the experimental result of 70.9 $\mathrm{meV}^{17}$. For the arsenic donors, the PBE extrapolated value is $22.5 \mathrm{meV}$, compared to an experimental value of $53.8 \mathrm{meV}^{17}$.

This underestimation of the binding energy can be attributed to the excess delocalization of the wavefunctions inherent in PBE, which results in a lower spin density close to the impurity site and thus a lower Coulomb attraction between the electron and the donor nucleus. This effect has been found in previous work on shallow impurities using $\mathrm{DFT}^{35-38}$. A better description of localization can be achieved with more sophisticated functionals, such as the hybrid functional of Heyd, Scuseria, and Ernzerhof $(\mathrm{HSE})^{42,43}$. Due to the vastly greater computational cost of HSE, we had to restrict ourselves to calculations up to $n=5(N=1000)$. We also note that computational limitations based on the size of the supercell prevented the calculation of the $\Delta V$ term for $n=7$, so PBE data is only reported up to $n=6$.

As we can see from the PBE results, results obtained with the $N=1000(n=5)$ supercells are not quite converged; however, the PBE results also show that $N \geq 512(n \geq 4)$ is within the regime in which the value of the hyperfine parameter $A$ scales as $1 / N$. We find that the PBE slope, suitably modified to take into account the PBE underestimation of the exchange splitting (as discussed in 
Table 1. Calculated parameters of shallow donors.

\begin{tabular}{|c|c|c|c|c|c|}
\hline$n$ & $\epsilon_{\Gamma}^{\mathrm{CB}}$ & $\epsilon_{\Gamma}^{\text {donor }}$ & $e \Delta V$ & $\delta^{\mathrm{ex}} / 2$ & $E_{\mathrm{b}}$ \\
\hline \multicolumn{6}{|c|}{$B i, P B E$} \\
\hline 2 & 6.415 & 6.082 & 0.109 & 0.0779 & 0.442 \\
\hline 3 & 6.392 & 6.206 & 0.052 & 0.0306 & 0.2 \\
\hline 4 & 6.283 & 6.206 & 0.029 & 0.0135 & 0.10 \\
\hline 5 & 6.251 & 6.202 & 0.019 & 0.0073 & 0.068 \\
\hline 6 & 6.243 & 6.206 & 0.014 & 0.0050 & 0.05 \\
\hline \multicolumn{6}{|c|}{$B i, H S E$} \\
\hline 2 & 6.825 & 6.266 & 0.081 & 0.2021 & 0.640 \\
\hline 3 & 6.781 & 6.503 & 0.027 & 0.0813 & 0.30 \\
\hline 4 & 6.669 & 6.544 & 0.021 & 0.0388 & 0.145 \\
\hline 5 & 6.650 & 6.557 & 0.023 & 0.0210 & 0.11 \\
\hline \multicolumn{6}{|c|}{$A s, P B E$} \\
\hline 2 & 6.415 & 6.023 & 0.009 & 0.0720 & 0.402 \\
\hline 3 & 6.392 & 6.205 & 0.007 & 0.0266 & 0.193 \\
\hline 4 & 6.283 & 6.212 & 0.013 & 0.0102 & 0.0 \\
\hline 5 & 6.251 & 6.210 & 0.014 & 0.0051 & 0.055 \\
\hline 6 & 6.243 & 6.214 & 0.011 & 0.0032 & $0.04 C$ \\
\hline \multicolumn{6}{|c|}{ As, HSE } \\
\hline 3 & 6.781 & 6.512 & 0.013 & 0.0750 & 0.282 \\
\hline 4 & 6.669 & 6.560 & 0.010 & 0.0311 & 0.119 \\
\hline 5 & 6.650 & 6.570 & 0.016 & 0.0156 & 0.0 \\
\hline
\end{tabular}

Quantities (in eV) used to calculate the shallow donor binding energy (Eq (1)), including half the exchange splitting $\delta^{\mathrm{ex}}$, which converges differently as a function of supercell size in PBE and HSE (see Supplementary Fig. 1).

the Supplementary Methods and shown in Supplementary Fig. 1), can be used to extrapolate the HSE data to the limit $N \rightarrow \infty$. Extrapolating the $n=5$ HSE data based on this slope gives the final prediction. This procedure gives $66.7 \mathrm{meV}$ for $\mathrm{Bi}$ donors and $53.9 \mathrm{meV}$ for As donors, in excellent agreement with the experimental values (70.9 meV for $\mathrm{Bi}$ and $53.8 \mathrm{meV}$ for $\mathrm{As})^{17}$. These results show that HSE is able to correct the PBE underestimation of shallow donor binding energies in silicon, while the limitation of HSE supercell size may be corrected by the PBE scaling, achieving remarkable agreement with experiment.

Hyperfine and superhyperfine parameters

The isotropic hyperfine parameter (in SI units) is given by:

$A=\frac{2 \mu_{0}}{3} g_{\mathrm{e}} \mu_{\mathrm{B}} g_{\mathrm{l}} \mu_{\mathrm{N}} \int \delta_{\mathrm{T}}(\mathbf{r}-\mathbf{R}) \sigma(\mathbf{r}) \mathrm{d} \mathbf{r}$,

where $g_{\mathrm{e}}$ is the electron $g$-factor, $g_{\mathrm{l}}$ is the $g$-factor of the nucleus in question, $\mu_{\mathrm{B}}$ is the Bohr magneton, $\mu_{\mathrm{N}}$ is the nuclear magneton, $\mathbf{R}$ is the position of the nucleus, and $\sigma(\mathbf{r})$ is the spin density. In the non-relativistic case discussed in ref. ${ }^{27}, \delta_{\mathrm{T}}$ is a Dirac delta function, but here a more extended function is used to take relativistic effects into account ${ }^{28,44}$.

Results for the hyperfine parameter of bismuth and arsenic shallow donors are shown in Fig. 2. The error is expected to scale inversely with the volume of the supercell much as in the case of binding energies, so we again plot our results as a function of $1 / \mathrm{N}$. The linear trend in hyperfine parameter for PBE calculations starts at even lower cell size of $n \geq 3(N \geq 216)$ than the binding energies, allowing extrapolation to the $N \rightarrow \infty$ limit. Extrapolating PBE results gives a value of $891 \mathrm{MHz}$ for bismuth donors, significantly underestimating the experimental result of $1475 \mathrm{MHz}^{22}$. For the arsenic donors, the extrapolated value is $93.6 \mathrm{MHz}$, compared to an experimental value of $198.3 \mathrm{MHz}^{17}$.

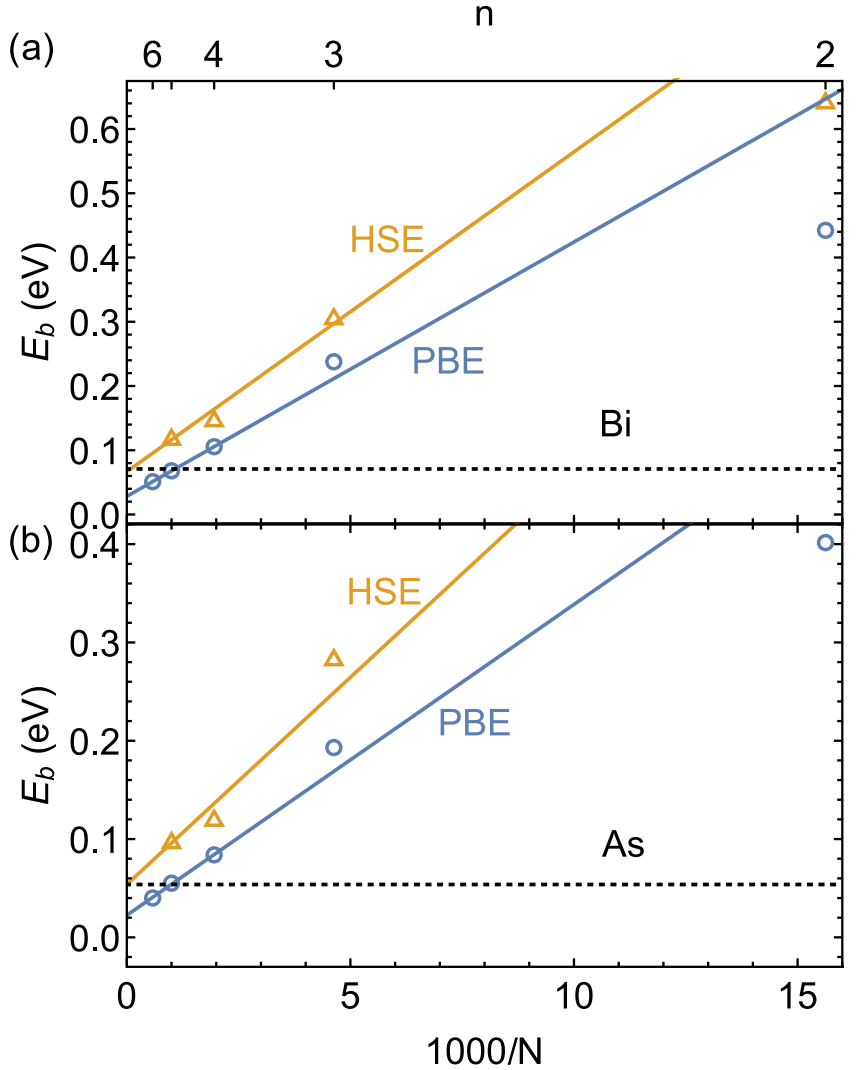

Fig. 1 Binding energies for donors in silicon as a function of supercell size. Binding energy is defined by Eq. (1). PBE results are in blue circles and HSE results are in orange triangles. The horizontal axis is $1 / N$, where $N$ is the number of atoms in the supercell. a Bismuth donor. The blue line is a linear fit to the PBE data for $n \geq 4$, $N \geq 512: E_{\mathrm{b}}=0.0283+39.6 / N$. The HSE slope is calculated by correcting for the difference in PBE and HSE exchange splitting (see Supplementary Methods and Supplementary Fig. 1). This slope is then applied to the HSE data with $n=5, E_{\mathrm{b}}=0.0667+49.7 / N$. The HSE extrapolated value of $66.7 \mathrm{meV}$ is in very good agreement with the experimental binding energy of $70.9 \mathrm{meV}$, shown as a black dashed line. b Arsenic donor. Blue PBE line fit to data for $n \geq 4, N \geq$ 512 is $E_{\mathrm{b}}=0.0225+31.6 / \mathrm{N}$, and the HSE line is $E_{\mathrm{b}}=0.0539+42.1 /$ $N$. This HSE extrapolated value of $53.9 \mathrm{meV}$ is in extremely good agreement with the experimental binding energy of $53.8 \mathrm{meV}$.

This underestimation of the hyperfine parameter is again due to a lack of localization in the PBE wavefunction, and can be improved using HSE in a similar way as for the binding energies. HSE results show linear scaling in the same regime as PBE and with the same slope, supporting our assertion that PBE scaling extends to HSE results for binding energies. This procedure gives $1262 \mathrm{MHz}$ for Bi donors and $132.5 \mathrm{MHz}$ for As donors (compared with experimental values of $1475 \mathrm{MHz}$ for $\mathrm{Bi}^{22}$ and $198.3 \mathrm{MHz}$ for $\left.A s^{17}\right)$, providing a vast improvement over PBE.

In addition to the Fermi contact interaction with the donor nucleus, the spin density induced by the donor also leads to socalled "superhyperfine" (shf) interactions with any ${ }^{29} \mathrm{Si}$ nuclei in the host ${ }^{22,33}$. We calculate shf parameters for various shells of $\mathrm{Si}$ atoms using a similar procedure as for the hyperfine parameter $A$ [Eq. (2); see Supplementary Notes and Supplementary Fig. 2]. The results are displayed in Fig. 3. Because the spin density does not decrease monotonically with distance from the donor ("Kohn-Luttinger oscillations"14,33), the shf parameter peaks at the 4th-nearest-neighbor $[A(004)]$ shell. This is reflected in our calculations (Fig. 3), which are in reasonable agreement with experiment and have comparable accuracy to earlier work using the Green's function method ${ }^{32}$. This provides further evidence 


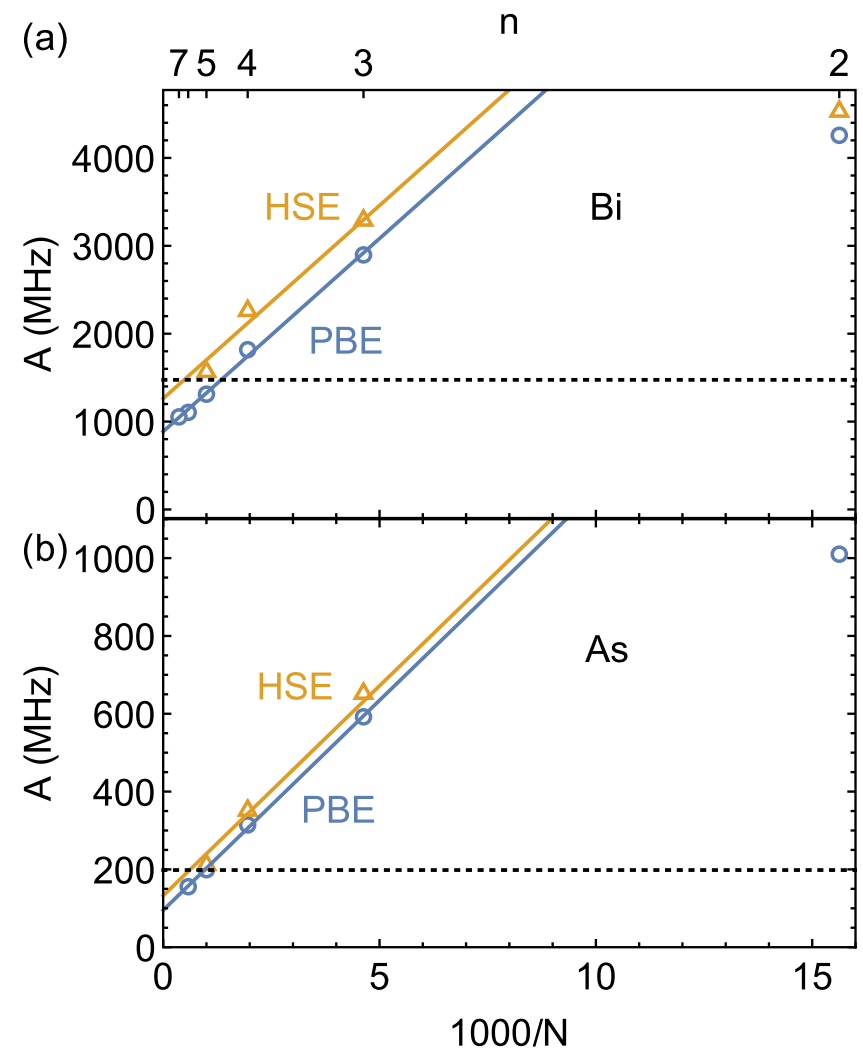

Fig. 2 Isotropic hyperfine parameter for donors in silicon as a function of supercell size. PBE results are in blue and HSE results are in orange. a Bismuth donor. The blue line shows a linear fit to PBE data for $n \geq 3, N \geq 216: A=887+4.39 \times 10^{5} / N$. The orange line has a slope fixed to the PBE value and intercept fit to the HSE data for $n \geq$ 3, $N \geq 216: A=1263+4.39 \times 10^{5} / \mathrm{N}$. The dashed black line is the experimental value, $A=1475 \mathrm{MHz}$. b Arsenic donor, with the same color coding as a. PBE fit: $A=95.2+1.08 \times 10^{5} / \mathrm{N}$. Line with slope fixed by the PBE fit and intercept fit to HSE data for $n \geq 3: A=$ $132.5+1.08 \times 10^{5} / \mathrm{N}$. The experimental value is $A=198.3 \mathrm{MHz}$.

that our approach is able to accurately describe the shallow-donor spin density.

Electric field gradients and quadrupole couplings

Our ability to model shallow donors using first-principles methods allows us to address unexpected strain-induced spin resonance splittings first observed in the experiments by Pla et al. ${ }^{22}$ described in the Introduction. We want to identify the mechanism by which strain causes a dramatic splitting in the ESR lines for $\mathrm{Bi}$ donors in Si. Traditionally, strain effects have been explained through the "valley repopulation model"25, which describes changes in the valley-orbit ground state caused by shifts of the conduction-band valleys. This model provides no explanation for the observed ESR splittings.

Pla et al. ${ }^{22}$ therefore explored other potential mechanisms for the strain dependence of the ESR splittings. One of these mechanisms is based on a term in the ESR Hamiltonian, which couples the quadrupole moment of the donor nucleus with the electric field gradient (EFG) induced by the electron wavefunction. This model includes a parameter $\gamma$, which enters as a scaling term in the quadrupole Hamiltonian. The main contribution to this term should be the Sternheimer antishielding factor, a measure of how the core electrons amplify an externally applied EFG (in this case coming from the shallow donor wavefunction). Pla et al. ${ }^{22}$ found that $\gamma=-900$ would be required in this model to fit their data, close to the value for isolated $\mathrm{Bi}^{+}$ions $(\gamma=-925.6)$. However, it is

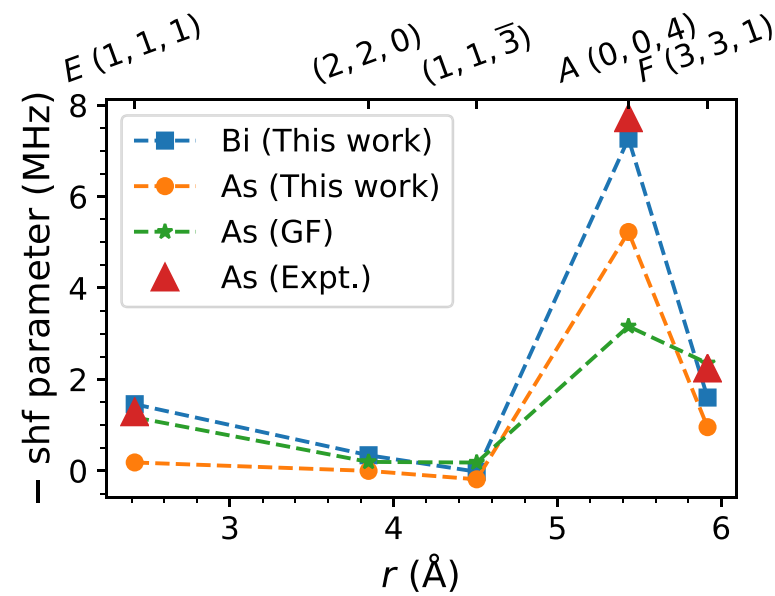

Fig. 3 Superhyperfine (shf) parameters as a function of distance to the donor center. Shf parameters for ${ }^{29} \mathrm{Si}$ in various shells surrounding the $\mathrm{Bi}$ donor are shown in blue squares, and shells surrounding the As donor are plotted as orange circles. We plot the negative of the shf parameter because the ${ }^{29} \mathrm{Si} g$ factor is negative. The predicted values are extrapolated to the $N \rightarrow \infty$ limit in a similar way to the hyperfine parameter, though larger supercells are required to see $1 / \mathrm{N}$ scaling (see Supplementary Fig. 2). Top axis ticks identify the neighbor shells, including the alphabetic labels from refs. ${ }^{32,57}$. Shown for comparison are experimental values from ref. ${ }^{57}$ (red triangles for the $E, A$, and $F$ shells; the other shells were not identified) and theoretical values from the Green's Function (GF) method of ref. ${ }^{32}$ (green stars). Dotted lines connecting the theory results are a guide to the eye.

not clear whether this value of $\gamma$ is realistic for a $\mathrm{Bi}$ donor in $\mathrm{Si}$. Other observations, such as the variation in strain splittings seen for different ESR transitions, are also not well explained by the quadrupole mechanism ${ }^{22}$

Being able to calculate the EFG from first principles would clearly shed light on this issue. Ab initio calculations using the projector-augmented-wave method have successfully determined EFGs at nuclei without using empirical corrections such as the Sternheimer antishielding factor ${ }^{26}$. An accurate calculation of the donor wavefunction will thus allow the determination of the EFG at the nucleus and the strength of the quadrupole coupling.

We have calculated the EFG at the nucleus of the Bi shallow donor in unstrained silicon and find it to be zero to within the accuracy of our calculations. We estimate this error bar to be approximately $1 \mathrm{~V} / \AA^{2}$, corresponding to a quadrupole interaction strength of approximately $1 \mathrm{MHz}$. For the unstrained case, the zero value is as expected, since symmetry arguments show that the EFG should vanish ${ }^{22}$. However, the calculated EFGs remain zero (within the error bar) when strain is applied. This runs counter to the quadrupole mechanism considered, amongst others, in Pla et al. 22 , as this required a quadrupole interaction on the order of $100 \mathrm{MHz}$. This result would appear to rule out the quadrupole splitting as one of the potential mechanisms for the strain-induced splittings observed in ESR.

These results highlight the need for a different mechanism to explain the observed splitting. In the next section, we will explore the shift of the isotropic hyperfine parameter with the hydrostatic component of strain.

\section{Strain}

We now address how the splittings of the ESR lines observed in the refs. ${ }^{7,22}$ can be explained by the strain dependence of the isotropic hyperfine parameter $A$. Symmetry restricts the form of the strain dependence of the isotropic hyperfine parameter ${ }^{7}$. 
Expanded to second order in strain, this dependence is given by

$$
\begin{aligned}
A / A_{0}= & 1+\frac{K}{3}\left(\epsilon_{\mathrm{xx}}+\epsilon_{\mathrm{yy}}+\epsilon_{\mathrm{zz}}\right) \\
& +\frac{L}{2}\left[\left(\epsilon_{\mathrm{yy}}-\epsilon_{\mathrm{zz}}\right)^{2}+\left(\epsilon_{\mathrm{yy}}-\epsilon_{\mathrm{zz}}\right)^{2}+\left(\epsilon_{\mathrm{yy}}-\epsilon_{\mathrm{zz}}\right)^{2}\right],
\end{aligned}
$$

where $A_{0}$ is the value in the absence of strain. Shear terms can be shown to be negligible ${ }^{7}$ and are not discussed here. The parameters $K$ and $L$ can be obtained by fitting to experimental results ${ }^{7,22}$. To pinpoint the underlying mechanisms of the ESR line splittings, the $K$ and $L$ values calculated for these mechanisms need to agree with experiment.

The common assumption is that the variation in $A$ would be attributed to the non-hydrostatic component of strain; this is referred to as the "valley repopulation model" 25 and requires an assessment of the parameter $L$. $L$ can be estimated as $L=-2 \Xi_{u}^{2} /\left(9 \Delta^{2}\right)$, where $\Xi_{u}=8.6 \mathrm{eV}$ is the uniaxial deformation potential of the CBM and $\Delta$ is the splitting between the $A_{1}$ and the $E$ valley-orbit states ${ }^{7,25}$. This estimate results in $L=-9720$, in good agreement with $L=-9800 \pm 2100$ fit to experimental data and $L=-9064$ extracted from tight-binding theory ${ }^{7,22}$.

However, the quadratic dependence of $A$ on nonhydrostatic strain implies that it is only ever reduced from the unstrained value, while the experimental results require both positive and negative contributions; i.e., the hydrostatic component, proportional to the parameter $K$, is playing an important role. Since hydrostatic strain acts on all the valleys equally, it does not lead to a repopulation effect. However, the magnitude of the wavefunction at the donor nucleus-which determines $A$-will be affected by hydrostatic strain, leading to a nonzero value of $K$.

The traditional assessment of this dependence on hydrostatic strain is based on effective mass theory, in which the dependence occurs through variations in the dielectric constant $\varepsilon$ and effective mass $m^{*}$. The donor Bohr radius $a_{\mathrm{D}}$ is proportional to $\varepsilon / m^{* 12}$. Literature shows that, for small strains, these properties vary linearly as a function of hydrostatic pressure. According to ref. ${ }^{45}$, $\Delta \varepsilon / \varepsilon=-2.8 \times 10^{-3}$ for $P=1 \mathrm{GPa}$, and according to ref. ${ }^{46}, \Delta \mathrm{m}^{*}$ / $m^{*}=-1.4 \times 10^{-3}$ for $P=1800 \mathrm{~kg} / \mathrm{cm}^{2}$. Using the bulk modulus of silicon $(97.88 \mathrm{GPa})^{47}$, these results give the dependence of $\varepsilon$ and $m^{*}$ on hydrostatic strain $\epsilon_{\mathrm{xx}}=\epsilon_{\mathrm{yy}}=\epsilon_{\mathrm{zz}}=\epsilon$ :

$\frac{\Delta \varepsilon}{\varepsilon}=0.819 \epsilon$ and $\frac{\Delta m^{*}}{m^{*}}=2.322 \epsilon$.

The donor Bohr radius is therefore given by

$a_{\mathrm{D}}(\epsilon)=a_{\mathrm{D} 0} \frac{1+0.819 \epsilon}{1+2.322 \epsilon}$.

The effective-mass-theory wavefunction is proportional to $a_{\mathrm{D}}^{-3 / 2}$ and the hyperfine parameter is proportional to the square of the wavefunction at the nucleus (Eq. (2)) ${ }^{12}$. Therefore the hyperfine parameter is

$A(\epsilon)=A_{0} \frac{(1+2.322 \epsilon)^{3}}{(1+0.819 \epsilon)^{3}}=A_{0}\left[1+4.524 \epsilon+\mathcal{O}\left(\epsilon^{2}\right)\right]$.

In the notation of Eq. (3), this corresponds to $K=4.524$. The experimentally observed value is $K=19.1^{22}$. Based on effective mass theory, we would thus conclude that impact of hydrostatic strain on the hyperfine parameter is much too small to explain the experimental splittings. But is effective mass theory sufficiently accurate? We have already seen that central cell effects are key to the accuracy of our approach. The issue became particularly relevant in light of subsequent experiments ${ }^{7}$, that confirmed the unexpectedly large dependence of the isotropic hyperfine parameter $A$ on the hydrostatic component of strain. A very informative treatment of the shortcomings of effective mass theory and how they can be overcome in first-principles calculations was provided by Huebl et al. in ref. ${ }^{33}$. They showed that, for $\mathrm{P}$ donors in strained silicon, valley repopulation theory is not adequate to describe the change in hyperfine parameter. They
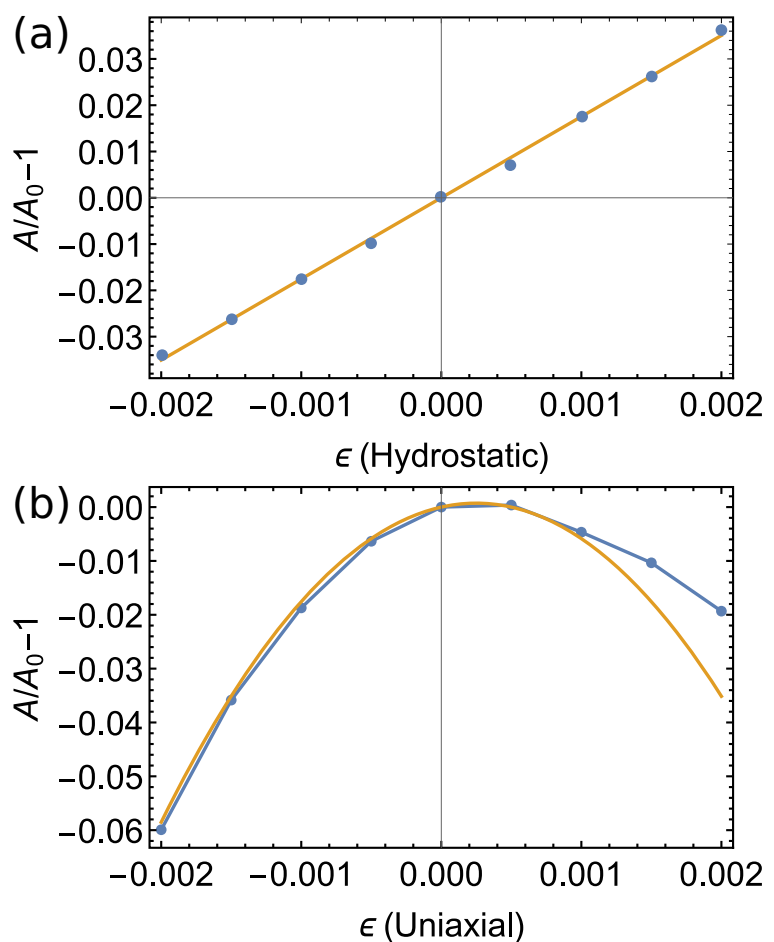

Fig. 4 Hyperfine parameter versus strain. $A / A_{0}-1$ is plotted versus strain for a bismuth donor in silicon in the $n=6, N=1728$-atom supercell using PBE. a Hydrostatic strain. A linear fit (orange line) produces $K=17.5$ (Eq. (3)). b Uniaxial strain. Taking $K=17.5$ fixed based on the hydrostatic case, a fit to the data up to $\epsilon=10^{-3}$ using Eq. (3) is shown (orange line), which gives $L=-11,700$.

were able to achieve good agreement with experiment by performing $a b$ initio calculations within the Green's function method.

We will therefore use our first-principles approach to explicitly explore the strain dependence for Bi donors. The calculated dependence of the isotropic hyperfine parameter is shown in Fig. 4 for a $n=6(N=1728)$ supercell using PBE; the behavior as a function of supercell size and functional will be discussed below. Figure 4a shows the data for hydrostatic strain. In each case the atomic structure was fully relaxed. The bismuth-silicon bond lengths in the unstrained case are $2.651 \AA$, compared to $2.367 \AA$ in bulk silicon. When hydrostatic strain is applied, all $\mathrm{Bi}-\mathrm{Si}$ and $\mathrm{Si}-\mathrm{Si}$ bond lengths simply scale with the hydrostatic strain to within better than $0.001 \AA$. The values of $A / A_{0}-1$ in Fig. 4 are well described by a linear fit up to the highest strains tested $\left(2 \times 10^{-3}\right)$, with a coefficient that corresponds to $K=17.5$ (Eq. (3)). This value is in good agreement with the experimental value $K=19.1^{7}$.

Figure $4 \mathrm{~b}$ shows the results for uniaxial strain. We varied $\epsilon_{\mathrm{zz}}$ up to $2 \times 10^{-3}$, keeping $\epsilon_{\mathrm{xx}}=\epsilon_{\mathrm{yy}}=0$. The calculated points are fitted to Eq. (3) using the $K$ value obtained from the hydrostatic strain calculations. Deviations from the quadratic behavior are observed for higher strains, consistent with the higher-order repopulation effects. The fit results in $L=-11,700$, in reasonable agreement with the values $L=-9720$ predicted by the valley repopulation model and $L=-9064$ calculated from tight-binding theory ${ }^{7,22}$. The reason for the decrease in $L$ with uniaxial strain will be discussed in the subsection on "Irreducible representations".

We now discuss the sensitivity of the strain results to supercell size and choice of functional. The calculation of the hyperfine parameter as a function of hydrostatic strain [Fig. 4a for $n=6, N=$ 1728] was repeated at different supercell sizes and subjected to similar scaling analysis as binding energies (Fig. 1) and hyperfine parameters (Fig. 2). The results of this analysis are shown in Fig. $5 . K$ 
$\mathrm{n}$

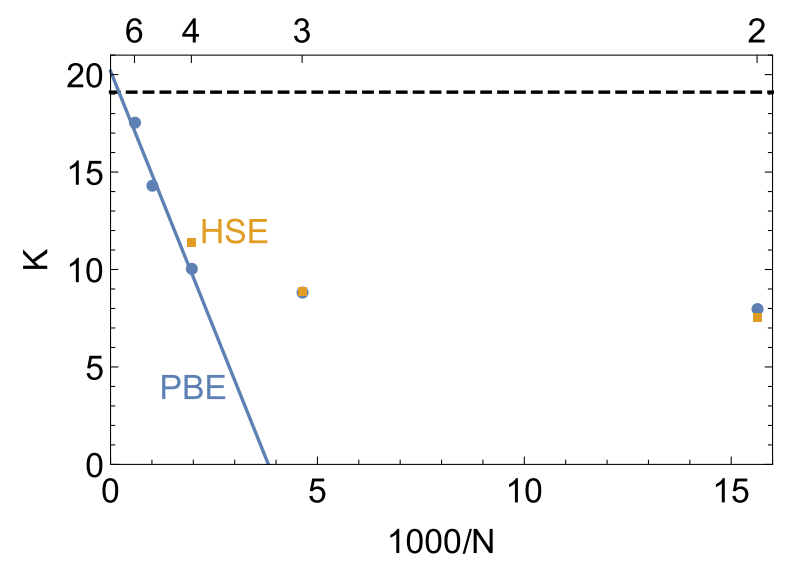

Fig. 5 Calculated $K$ coefficient as a function of supercell size. $K$ is defined by Eq. (3). Data is for bismuth donors, with PBE in blue circles and HSE in orange squares. A fit to PBE data for $N \geq 512$ is shown: $K=20.2-5290 / N$. The dashed line shows the experimental value.

also shows linear scaling with $1 / N$, but it begins at a higher $N$ value (corresponding to $n=4$ ) than observed for the hyperfine parameter itself. Extrapolation to the dilute limit gives a coefficient of $K=20.2$, in good agreement with the experimental value $K=19.1^{7}$.

As to the influence of the functional, as shown in Fig. 5 the HSE values for $n=2,3$, and 4 are very close to the PBE results. We therefore feel that the PBE results can be considered accurate to within an error bar of \pm 1 . We thus find that, while the $A$ parameter itself requires a calculation using an advanced functional such as $\mathrm{HSE}$, its variation with strain (which presumably is determined by a redistribution of the wavefunction as a function of volume) is adequately described with PBE.

Our results clearly show that the isotropic hyperfine parameter depends linearly on the hydrostatic component of strain with a coefficient that significantly differs from that predicted by effective mass theory, highlighting the importance of valleyorbit coupling and central-cell corrections.

\section{Irreducible representations}

The symmetry and group-theoretic properties of the ground-state wavefunction are key to all of our results. As discussed in the "Introduction" section, the 6-fold degenerate CBM splits into states corresponding to irreducible representations (irreps) of the $T_{\mathrm{d}}$

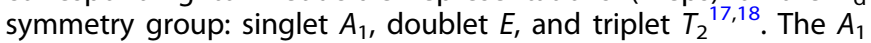
wavefunction (the fully symmetric state) has a peak at the center while the others have nodes. This allows the $A_{1}$ state to maximize the Coulomb binding energy, making it the ground state, with an energy that is significantly lowered compared to effective mass theory. This also means that the hyperfine parameter is directly related to the amount of $A_{1}$ character in the ground state; as more of the other irreps are mixed in, the hyperfine parameter decreases. In the case of uniaxial strain (Fig. 4b), the symmetry is lowered, at the expense of the weight of the fully symmetric $A_{1}$ state, and $A$ decreases for both compressive and expansive uniaxial strain.

To demonstrate that our calculations correctly capture these symmetry effects, we project out the portions of the donor wavefunctions which transform according to the various irreps of $T_{\mathrm{d}}$. We use the projection operator

$\hat{P}^{j}=\frac{l_{j}}{h} \sum_{R} X(R)^{j} \hat{R}$

where $j$ indexes the various irreps, $l_{j}$ is the dimension of the irrep, $h$ is the order of the $T_{\mathrm{d}}$ group, $R$ is summed over all the symmetry

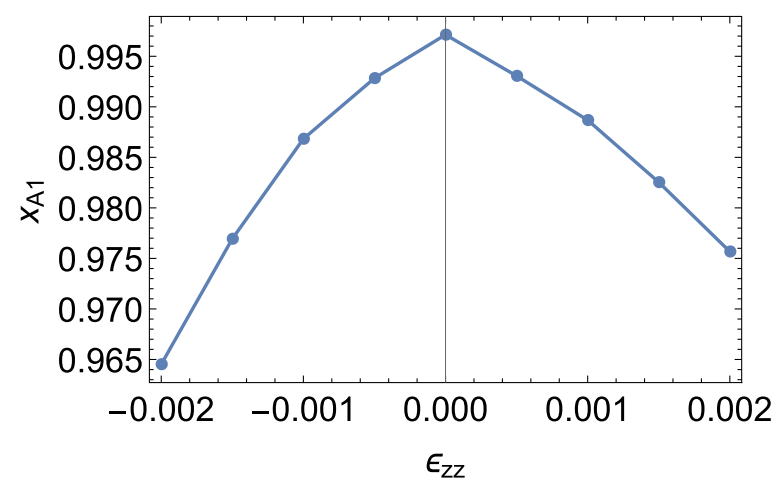

Fig. 6 Changes in symmetry with strain. Fraction of the bismuth donor spin density which transforms according to the $A_{1}$ irreducible representation (Eq. (8)), plotted as a function of applied uniaxial strain. At zero strain, $\rho$ transforms under the $A_{1}$ representation. As uniaxial strain is applied, the fraction of $\rho$ which transforms under $A_{1}$ decreases.

operations in the group, and $X(R)^{j}$ is the character of the operation in the $j$ th representation ${ }^{48}$. With cell volume $\Omega$, we define the fraction of the spin density $\rho$ which transforms under the jth irrep

$x_{\mathrm{j}}=\frac{1}{\Omega} \int d^{3} \mathbf{r} \frac{\left|\hat{P}^{j} \rho\right|}{|\rho|}$.

The $x_{\mathrm{A} 1}$ values for bismuth donors as a function of strain $\epsilon_{\mathrm{zz}}$ with $\epsilon_{\mathrm{xx}}=\epsilon_{\mathrm{yy}}=0$ are shown in Fig. 6. At zero strain, $\rho$ transforms almost entirely under the $A_{1}$ representation, and as uniaxial strain is applied, the fraction of $\rho$ which transforms under $A_{1}$ decreases, following a trend similar to the hyperfine parameter (Fig. 4b).

Visualizations of the projected spin density are shown in Fig. 7. The "Kohn-Luttinger oscillations" evident in the shf parameters (Fig. 3) may also be seen in the shape of the visualized spin density, which differs significantly from the effective-mass-theory prediction.

\section{DISCUSSION}

The study of shallow impurity wavefunctions has a long history, beginning with Kohn-Luttinger effective mass theory in the 1950s. We have overcome some of the challenges to modeling these wavefunctions from first principles, allowing us to employ DFT calculations which fully include central-cell and valley-orbit effects. This allows us to study the evolution of the hyperfine parameter and quadrupole coupling of shallow donors as a function of strain without the use of empirical fitting parameters.

We have calculated accurate hyperfine parameters, superhyperfine parameters, and binding energies of bismuth and arsenic shallow donors by using PBE, identifying a $1 / \mathrm{N}$ scaling with supercell size and extrapolating to the $N \rightarrow \infty$ limit, and correcting the results for PBE self-interaction error using the HSE hybrid functional. This represents a significant step forward in the ab initio simulation of shallow donors in silicon, and we expect it to be generalizable to other shallow impurities in silicon and other materials.

The predictive nature of our model allows us to explore other properties based on the calculated electron density. We find that the electric field gradients at the site of the nucleus are negligibly low, even when strain is applied. This suggests that the splittings observed by Pla et al. ${ }^{22}$ in ESR experiments on Bi donors in $\mathrm{Si}$ do not in fact arise from quadrupole interactions. Instead, our calculations lend strong support to the alternative mechanism explored in the ref. ${ }^{7}$, which suggests a large linear dependence of the isotropic hyperfine parameter $A$ on the hydrostatic component of strain. Our calculations find just such a dependence, with a 

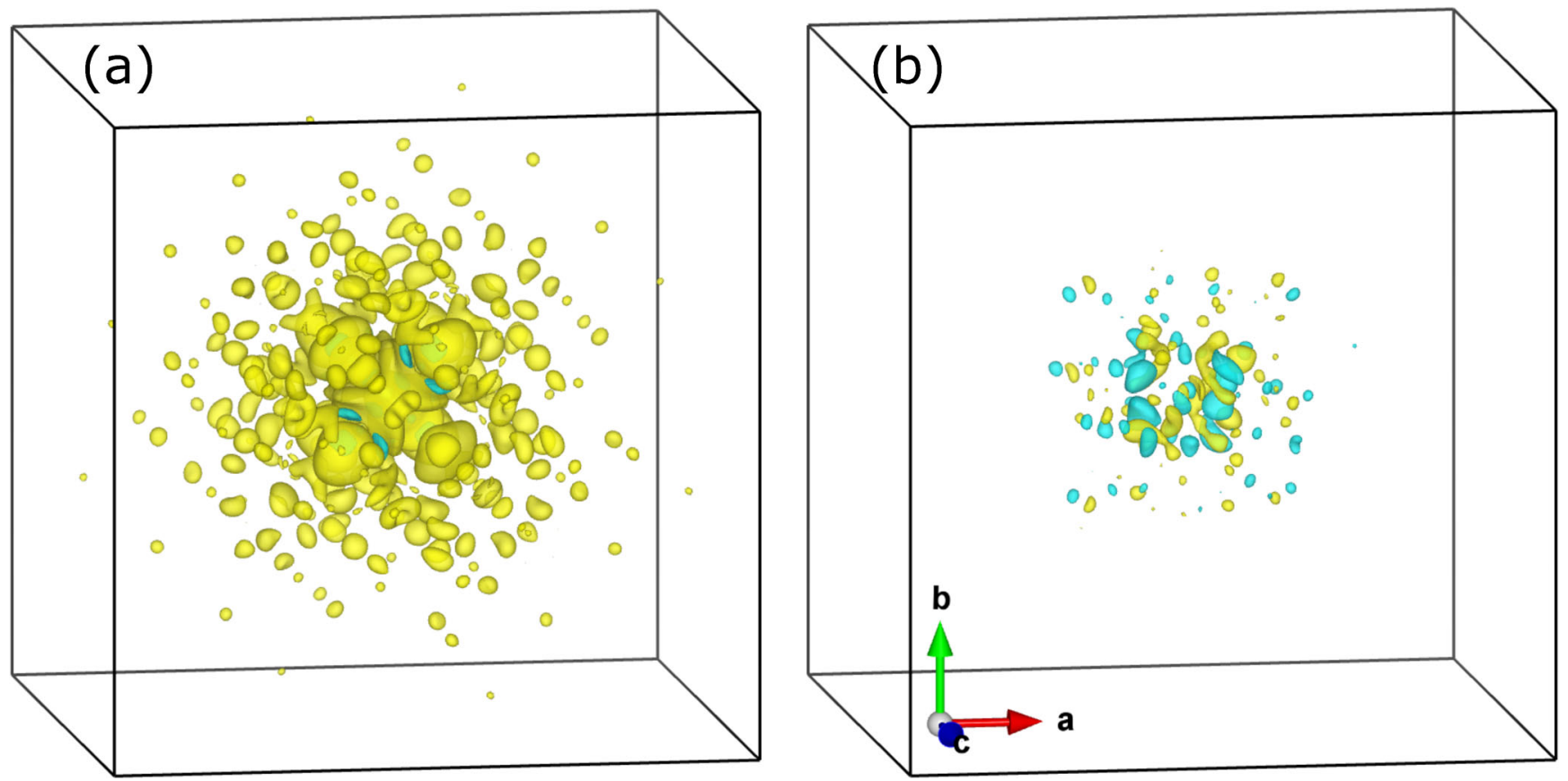

Fig. 7 Visualization of symmetry in the electron density. Results of projecting $\rho$ onto the $A_{1}$ representation for a bismuth donor calculation with $\epsilon_{\mathrm{xx}}=10^{-3}$ uniaxial strain, in a $N=1728(n=6)$ supercell. a The portion of $\rho$ which transforms as $A_{1}$. Isosurface value $2.7 \times 10^{-4} e / \AA^{3}$. b The remainder after projection. Isosurface value $5.4 \times 10^{-5} \mathrm{e} / \AA^{3}$.

coefficient $K=20.2$ that is in good agreement with the experimental value $K=19.1$. The linear dependence on hydrostatic strain is significantly larger than the dependence predicted by effective mass theory.

This accurate, predictive, and generalizable model represents an important step in improving the ab initio description of shallow donors and their hyperfine structure. These theoretical tools go hand-in-hand with experiments, and can be instrumental in the ongoing effort to develop silicon-based spin qubits.

\section{METHODS}

Density functional theory

We use spin-polarized DFT with the projector-augmented wave method ${ }^{49}$ in the Vienna Ab-initio Simulation Package (VASP) ${ }^{50}$. Calculations employed the Perdew-Burke-Ernzerhof (PBE) exchange-correlation functiona ${ }^{41}$ as well as the hybrid functional of Heyd, Scuseria, and Ernzerhof $(\mathrm{HSE})^{42,43}$ with the standard parameters. This predicts a band gap of 1.16 $\mathrm{eV}$, in very good agreement with the experimental zero-temperature gap of $1.17 \mathrm{eV}^{51}$, suggesting that this functional provides a good description of the electronic structure of silicon in agreement with previous work ${ }^{52}$. The PBE value is $0.63 \mathrm{eV}$, The HSE-predicted dielectric constant of 11.1 is in good agreement with the experimental value $11.7^{53}$, and the predicted lattice constant $5.433 \AA$ is in excellent agreement with the experimental $5.431 \AA^{54}$.

Hyperfine parameters were calculated following the methodology described in the refs. ${ }^{28,44,55,56}$. The core spin polarization is taken into account through the frozen valence approximation developed in the ref. ${ }^{56}$ and validated in the context of HSE in the ref. ${ }^{28}$. We use the VASP standard PAW pseudopotentials for the PBE functional, with $[\mathrm{Xe}] 4 \mathrm{f}^{14}$ in the bismuth core and $[\mathrm{Ne}] 3 \mathrm{~s}^{2} 3 \mathrm{p}^{6}$ in the arsenic core. Electric field gradients and quadrupole couplings were calculated using the method of ref. ${ }^{26}$. Calculations were performed in supercells that are $n \times n \times n$ multiples of the conventional 8-atom cubic cell. The largest PBE supercell corresponds to $n=7$, containing $N=2744$ atoms. The largest HSE supercell corresponds to $n=5$, containing $N=1000$ atoms. A plane-wave basis with an energy cutoff of $250 \mathrm{eV}$ was used and the supercells were relaxed so that all forces were smaller than $1 \mathrm{meV} / \AA$. Details on the computational cost of the hybrid functional calculations may be found in the Supplementary Discussion. Eigenvalue differences are converged to within
$0.8 \mathrm{meV}$ with respect to the cutoff, and hyperfine parameters are converged to within $2.5 \%$.

\section{Finite size effects}

The Bohr radius of a shallow donor in silicon within effective mass theory is approximately $23.8 \AA$. This is only slightly smaller than the side lengths of the largest $(n=7, N=2744)$ supercells we use: $38.3 \AA$. The Coulomb envelope only drops to $1 \%$ of its central value $54.7 \AA$ from the center. Therefore, a significant part of this exponential tail necessarily overlaps into neighboring supercells. We will therefore need to extrapolate to the $N \rightarrow \infty$ limit, where $N$ is the number of atoms in the supercell, in order to calculate physical results. For large enough supercells, the error scales inversely with the volume of the supercell, or equivalently with $1 / N$. This involves fitting lines to the data versus $1 / \mathrm{N}$. All fits are based on the linear least-squares method.

\section{Occupation of states}

Occupancies of the Kohn-Sham states in DFT are typically set through a "smearing" procedure. This is done to avoid various convergence issues that can arise when bands are partially occupied, and a scheme for extrapolating to zero smearing is typically included. This procedure is benign for most situations, but it leads to severe problems in this particular case. The energy spacing between valley-orbit split states of the donor wavefunction is approximately $10 \mathrm{meV}$, comparable in size to the smallest smearing parameters typically used; smearing will mix states other than the ground state into the final charge density. This is a problem because the $A_{1}$ state has a peak at the nucleus, while the other states have nodes. We are comparing to experiments performed at low temperature, so the measured properties are determined entirely by the ground state. Mixing with excited states of the donor will defeat any chance of calculating the hyperfine parameter correctly. To overcome this issue, we do not use smearing in our final calculations, fixing the spin-up occupancy of the lowest-energy conduction band to one. This requires care to ensure proper convergence, but has proven feasible through the use of intermediate calculations which include small amounts of smearing.

\section{Brillouin zone sampling}

The $A_{1}$ valley-orbit ground state is made up of a combination of all six conduction-band valley states. Therefore a correct calculation of the donor wavefunction must include contributions from each valley. For the 
equilibrium structure, and under hydrostatic strain, the six valleys are equivalent and equally occupied. Under uniaxial deformation, splitting of the valleys occurs, and ensuring correct sampling of all of the valleys (which is necessary to obtain the correct valley-orbit splitting) could be tricky.

Most of the supercells used in the present study are large enough to ensure that, in reciprocal space, the conduction-band valleys are folded back to a point very close to the zone center. Therefore sampling the Brillouin zone at a single $\mathbf{k}$ point automatically allows for all of the proper interactions and mixing between the valleys in the self-consistent calculation. Our calculations use the $\Gamma$ point to maintain an unbiased sampling of the valleys and to reduce the computational demand. A detailed analysis of Brillouin-zone sampling was included in the ref. ${ }^{38}$, and also reached the conclusion that sampling at the $\Gamma$ point provided reliable results.

We verified that our calculations capture the correct valley-orbit state through symmetry analysis of the wavefunction.

\section{DATA AVAILABILITY}

The data that support the findings of this study are available in the NOMAD respository at https://doi.org/10.17172/NOMAD/2020.10.21-1.

Received: 16 April 2020; Accepted: 23 October 2020;

Published online: 27 November 2020

\section{REFERENCES}

1. Arute, F. et al. Quantum supremacy using a programmable superconducting processor. Nature 574, 505-510 (2019).

2. National Academies of Sciences, Engineering, and Medicine. Quantum Computing: Progress and Prospects (The National Academies Press, Washington, DC, 2019).

3. Preskill, J. Quantum computing in the NISQ era and beyond. Quantum 2, 79 (2018).

4. Clarke, J. \& Wilhelm, F. K. Superconducting quantum bits. Nature 453, 1031-1042 (2008).

5. Nayak, C., Simon, S. H., Stern, A., Freedman, M. \& Das Sarma, S. Non-abelian anyons and topological quantum computation. Rev. Mod. Phys. 80, 1083-1159 (2008).

6. Friis, N. et al. Observation of entangled states of a fully controlled 20-qubit system. Phys. Rev. X 8, 021012 (2018).

7. Mansir, J. et al. Linear hyperfine tuning of donor spins in silicon using hydrostatic strain. Phys. Rev. Lett. 120, 167701 (2018).

8. Tyryshkin, A. M. et al. Electron spin coherence exceeding seconds in high-purity silicon. Nat. Mater. 11, 143-147 (2012).

9. Saeedi, K. et al. Room-temperature quantum bit storage exceeding 39 minutes using ionized donors in silicon-28. Science 342, 830-833 (2013).

10. $\mathrm{Pla}$, J. J. et al. High-fidelity readout and control of a nuclear spin qubit in silicon. Nature 496, 334-338 (2013).

11. Muhonen, J. T. et al. Quantifying the quantum gate fidelity of single-atom spin qubits in silicon by randomized benchmarking. J. Phys. Condens. Matter 27, 154205 (2015).

12. Grundmann, M. The Physics of Semiconductors (Springer, New York, 2010).

13. Luttinger, J. M. \& Kohn, W. Motion of electrons and holes in perturbed periodic fields. Phys. Rev. 97, 869-883 (1955).

14. Kohn, W. \& Luttinger, J. M. Theory of donor states in silicon. Phys. Rev. 98, 915-922 (1955).

15. Kohn, W. \& Luttinger, J. M. Hyperfine splitting of donor states in silicon. Phys. Rev. 97, 883-888 (1955).

16. Kohn, W. Shallow impurity states in silicon and germanium. Solid State Phys. 5, 257-320 (1957).

17. Saraiva, A. L., Baena, A., Calderón, M. J. \& Koiller, B. Theory of one and two donors in silicon. J. Phys. Condens. Matter 27, 154208 (2015).

18. Wellard, C. J. \& Hollenberg, L. C. L. Donor electron wave functions for phosphorus in silicon: beyond effective-mass theory. Phys. Rev. B 72, 085202 (2005).

19. Nara, H. \& Morita, A. Shallow donor potential in silicon. J. Phys. Soc. Jpn 21, 1852-1853 (1966).

20. Baena, A., Saraiva, A. L., Koiller, B. \& Calderón, M. J. Impact of the valley degree of freedom on the control of donor electrons near a $\mathrm{Si} / \mathrm{SiO}_{2}$ interface. Phys. Rev. $B$ 86, 035317 (2012)

21. Gamble, J. K. et al. Multivalley effective mass theory simulation of donors in silicon. Phys. Rev. B 91, 235318 (2015).
22. Pla, J. J. et al. Strain-induced spin-resonance shifts in silicon devices. Phys. Rev. Appl. 9, 044014 (2018)

23. Wolfowicz, G. et al. Atomic clock transitions in silicon-based spin qubits. Nat. Nanotechnol. 8, 881 (2013).

24. Saeedi, K. et al. Optical pumping and readout of bismuth hyperfine states in silicon for atomic clock applications. Sci. Rep. 5, 10493 (2015).

25. Wilson, D. K. \& Feher, G. Electron spin resonance experiments on donors in silicon. III. investigation of excited states by the application of uniaxial stress and their importance in relaxation processes. Phys. Rev. 124, 1068-1083 (1961).

26. Petrilli, H. M., Blöchl, P. E., Blaha, P. \& Schwarz, K. Electric-field-gradient calculations using the projector augmented wave method. Phys. Rev. B 57, 14690-14697 (1998).

27. Van de Walle, C. G. Structural identification of hydrogen and muonium centers in silicon: first-principles calculations of hyperfine parameters. Phys. Rev. Lett. 64, 669-672 (1990).

28. Szász, K., Hornos, T., Marsman, M. \& Gali, A. Hyperfine coupling of point defects in semiconductors by hybrid density functional calculations: the role of core spin polarization. Phys. Rev. B 88, 075202 (2013).

29. Freysoldt, C. et al. First-principles calculations for point defects in solids. Rev. Mod. Phys. 86, 253 (2014).

30. Melnikov, D. V. \& Chelikowsky, J. R. Quantum confinement in phosphorus-doped silicon nanocrystals. Phys. Rev. Lett. 92, 046802 (2004).

31. Han, J., Chan, T.-L. \& Chelikowsky, J. R. Quantum confinement, core level shifts, and dopant segregation in p-doped Si<110〉 nanowires. Phys. Rev. B 82, 153413 (2010).

32. Overhof, H. \& Gerstmann, U. Ab initio calculation of hyperfine and superhyperfine interactions for shallow donors in semiconductors. Phys. Rev. Lett. 92, 087602 (2004).

33. Huebl, H. et al. Phosphorus donors in highly strained silicon. Phys. Rev. Lett. 97, 166402 (2006).

34. Gerstmann, U. Ab initio green's function calculation of hyperfine interactions for shallow defects in semiconductors. Phys. Status Solidi B 248, 1319-1336 (2011).

35. Wang, L.-W. Density functional calculations of shallow acceptor levels in Si. J. Appl. Phys. 105, 123712 (2009).

36. Zhang, G., Canning, A., Grønbech-Jensen, N., Derenzo, S. \& Wang, L.-W. Shallow impurity level calculations in semiconductors using ab initio methods. Phys. Rev. Lett. 110, 166404 (2013).

37. Yamamoto, T., Uda, T., Yamasaki, T. \& Ohno, T. First-principles supercell calculations for simulating a shallow donor state in Si. Phys. Lett. A 373, 3989-3993 (2009).

38. Smith, J. S. et al. Ab initio calculation of energy levels for phosphorus donors in silicon. Sci. Rep. 7, 6010 (2017).

39. Freysoldt, C., Neugebauer, J. \& Van de Walle, C. G. Fully ab initio finite-size corrections for charged-defect supercell calculations. Phys. Rev. Lett. 102, 016402 (2009).

40. Freysoldt, C., Neugebauer, J. \& Van de Walle, C. G. Electrostatic interactions between charged defects in supercells. Phys. Status Solidi 248, 1067-1076 (2011).

41. Perdew, J. P., Burke, K. \& Ernzerhof, M. Generalized gradient approximation made simple. Phys. Rev. Lett. 77, 3865-3868 (1996).

42. Heyd, J., Scuseria, G. E. \& Ernzerhof, M. Hybrid functionals based on a screened coulomb potential. J. Chem. Phys. 118, 8207-8215 (2003).

43. Paier, J. et al. Erratum: 'Screened hybrid density functionals applied to solids'. J. Chem. Phys. 125, 249901 (2006).

44. Blöchl, P. E. First-principles calculations of defects in oxygen-deficient silica exposed to hydrogen. Phys. Rev. B 62, 6158-6179 (2000).

45. Samara, G. A. Temperature and pressure dependences of the dielectric constants of semiconductors. Phys. Rev. B 27, 3494-3505 (1983).

46. Hensel, J. C., Hasegawa, H. \& Nakayama, M. Cyclotron resonance in uniaxially stressed silicon. II. Nature of the covalent bond. Phys. Rev. 138, A225-A238 (1965).

47. McSkimin, H. J. \& Andreatch, Jr, P. Elastic moduli of silicon vs hydrostatic pressure at $25.0^{\circ} \mathrm{C}$ and $-195.8^{\circ} \mathrm{C}$. J. Appl. Phys. 35, 2161-2165 (1964).

48. Cotton, F. A. Chemical Applications of Group Theory, 3rd edn. (Wiley, Hoboken, 1990).

49. Blöchl, P. E. Projector augmented-wave method. Phys. Rev. B 50, 17953-17979 (1994).

50. Kresse, G. \& Furthmüller, J. Efficient iterative schemes for ab initio total-energy calculations using a plane-wave basis set. Phys. Rev. B 54, 11169-11186 (1996).

51. Madelung, O., Rössler, U. \& Schulz, M. (eds). Silicon (Si), Band structure: datasheet from Landolt-Börnstein-Group III condensed matter · Volume 41A1 $\beta$ : "Group IV Elements, IV-IV and III-V Compounds. Part b-Electronic, transport, optical and other properties" (Springer, Berlin Heidelberg, 2002).

52. Hummer, K., Harl, J. \& Kresse, G. Heyd-Scuseria-Ernzerhof hybrid functional for calculating the lattice dynamics of semiconductors. Phys. Rev. B 80, 115205 (2009).

53. Dunlap, W. C. \& Watters, R. L. Direct measurement of the dielectric constants of silicon and germanium. Phys. Rev. 92, 1396-1397 (1953). 
54. Einspruch, N. VLSI Handbook. Handbooks in Science and Technology (Elsevier Science, Amsterdam, 2012).

55. Van de Walle, C. G. \& Blöchl, P. E. First-principles calculations of hyperfine parameters. Phys. Rev. B 47, 4244 (1993).

56. Yazyev, O. V., Tavernelli, I., Helm, L. \& Röthlisberger, U. Core spin-polarization correction in pseudopotential-based electronic structure calculations. Phys. Rev. $B$ 71, 115110 (2005).

57. Hale, E. B. \& Mieher, R. L. Shallow donor electrons in silicon. I. hyperfine interactions from ENDOR measurements. Phys. Rev. 184, 739-750 (1969).

\section{ACKNOWLEDGEMENTS}

Thanks to Pierandrea Conti at UCL for additional analysis of experimental data from ref. ${ }^{7}$. This work was supported in part by the UC Santa Barbara Quantum Foundry through the National Science Foundation "Quantum Materials Science, Engineering and Information (Q-AMASE-i)" program, Award \# DMR-1906325. M.W.S.'s work on superhyperfine parameters and exchange splitting was supported by an American Society for Engineering Education (ASEE) fellowship at the US Naval Research Laboratory. Use was made of computational facilities purchased with funds from NSF (CNS-1725797) and administered by the Center for Scientific Computing (CSC). The CSC is supported by the California NanoSystems Institute and the Materials Research Science and Engineering Center (NSF DMR-1720256) at UC Santa Barbara. This work also used the Extreme Science and Engineering Discovery Environment (XSEDE), which is supported by NSF grant number ACl-1548562, and the National Energy Research Scientific Computing Center, a DOE Office of Science User Facility supported by the Office of Science of the $U$. S. Department of Energy under Contract No. DE-AC02-05CH11231.

\section{AUTHOR CONTRIBUTIONS}

M.W.S., J.J.L.M., and C.G.V. designed the study; C.G.V. secured funding and computational resources; M.W.S., H.P., and S.M. performed the calculations and analysis; J.J.L.M. provided experimental data and analysis; M.W.S. and C.G.V. wrote the first draft; and all authors revised and edited the manuscript.

\section{COMPETING INTERESTS}

The authors declare no competing interests.

\section{ADDITIONAL INFORMATION}

Supplementary information is available for this paper at https://doi.org/10.1038/ s41524-020-00448-7.

Correspondence and requests for materials should be addressed to C.G.V.d.W.

Reprints and permission information is available at http://www.nature.com/ reprints

Publisher's note Springer Nature remains neutral with regard to jurisdictional claims in published maps and institutional affiliations.

(i) Open Access This article is licensed under a Creative Commons Attribution 4.0 International License, which permits use, sharing, adaptation, distribution and reproduction in any medium or format, as long as you give appropriate credit to the original author(s) and the source, provide a link to the Creative Commons license, and indicate if changes were made. The images or other third party material in this article are included in the article's Creative Commons license, unless indicated otherwise in a credit line to the material. If material is not included in the article's Creative Commons license and your intended use is not permitted by statutory regulation or exceeds the permitted use, you will need to obtain permission directly from the copyright holder. To view a copy of this license, visit http://creativecommons. org/licenses/by/4.0/.

(c) The Author(s) 2020 